\title{
Adenocarcinoma de úraco: relato de dois casos
}

Primeira submissão em 24/07/12 Última submissão em 14/08/12 Aceito para publicação em 14/08/12 Publicado em 20/10/12

\author{
Urachal adenocarcinoma: a report of two cases
}

Maurício Figueiredo Lima e Marchese'; Hamilton de Souza Mourão'; Limírio Leal da Fonseca Filho'; Hugo Hipólyto ${ }^{3}$; Cristina Louzada Landman ${ }^{4}$

unitermos
Adenocarcinoma
Úraco
Bexiga
Tumor

\section{resumo}

O adenocarcinoma de úraco é uma neoplasia incomum associada a prognóstico reservado. Realiza-se estudo clínico e anatomopatológico de dois casos de adenocarcinoma de úraco: um homem de 63 anos de idade que apresentou recidiva tumoral após cistectomia parcial aberta e evolução desfavorável; e uma mulher de 55 anos, submetida à cistectomia parcial videolaparoscópica, que se encontra em seguimento livre de recidiva.

\section{abstract}

Urachal adenocarcinoma is an uncommon neoplasm associated with poor prognosis. A clinical and pathological study of two cases of urachal adenocarcinoma is presented: a 63-year-old man with tumoral recurrence after open partial cystectomy and unfavorable evolution; a 55-year-old woman who underwent laparoscopic partial cystectomy without recurrence in the follow up. key words

Adenocarcinoma

Urachus

Vesical tumour

\section{Introdução}

Adenocarcinomas de bexiga são raros e representam $0,5 \%$ a $2 \%$ de todos os tumores malignos vesicais. Classificam-se como primários da bexiga (ou adenocarcinomas não úraco), de úraco ou lesões metastáticas ${ }^{(10)}$. Compreendem menos de $0,5 \%$ dos tumores vesicais ${ }^{(6)}$ e correspondem a aproximadamente $10 \%$ de todos os adenocarcinomas da bexiga ${ }^{(10)}$.

O primeiro caso descrito na literatura médica foi em 1863 por Hue e Jacquine, sendo mais tarde traduzido e sumarizado por Sheldon et al. ${ }^{(8)}$.

\section{Relato dos casos}

\section{Caso clínico 1}

Paciente do sexo masculino, 63 anos de idade, com queixa de hematúria macroscópica, dor suprapúbica e sintomas irritativos do trato urinário inferior, como urgeincontinência e noctúria. Ex-tabagista, hipertenso, coronariopata e obesidade grau I (índice de massa corporal [IMC] 32,6 kg/m²). Ao exame físico apresentava-se com abdômen globoso, flácido, indolor, sem visceromegalias; genitália sem anormalidades; toque retal com próstata de aproximadamente $50 \mathrm{~g}$, sem nódulos.

1. Residência médica em Cirurgia Geral e Urologia pelo Instituto de Assistência Médica ao Servidor Público de São Paulo (IAMSPE); mestrando do Programa de Pós-graduação em Ciências da Saúde do IAMSPE.

2. Diretor do Serviço de Urologia do IAMSPE.

3. Médico assistente do Serviço de Urologia do IAMSPE.

4. Residência médica em Anatomia Patológica IAMSPE; médica assistente do Serviço de Anatomia Patológica do IAMSPE. 
Exames de tomografia computadorizada (TC) e ressonância nuclear magnética (RNM) de abdômen evidenciaram bexiga de boa repleção, de paredes espessadas e massa junto à cúpula vesical, em topografia de úraco, medindo $4,6 \times$ $5 \mathrm{~cm}$, com realce ao meio de contraste (Figura 1).

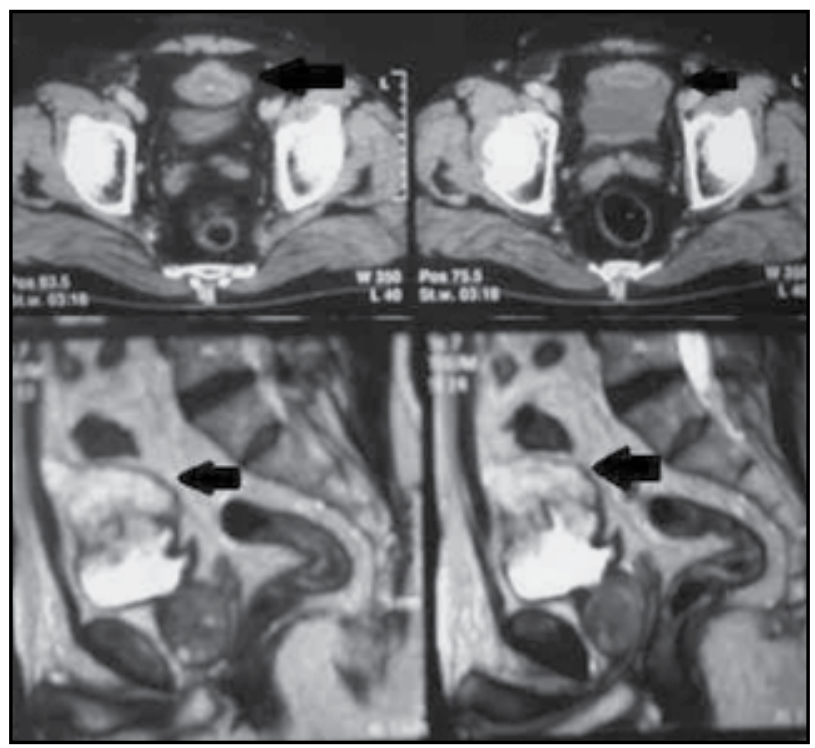

Figura 1 - Bexiga de paredes espessadas e massa junto à cúpula vesical em topografia de úraco (setas) com realce ao meio de contraste. Cortes axiais de TC acima e sagitais de RNM abaixo

TC: tomografia computadorizada; RNM: ressonância nuclear magnética.

O paciente foi submetido à cistoscopia, que evidenciou lesão sólida de aspecto indiferenciado, com cerca de $4 \mathrm{~cm}$, em cúpula, meatos ureterais tópicos e ejaculando urina clara; restante da mucosa vesical livre de lesões. Realizou-se ressecção transuretral (RTU) da lesão e o exame anatomopatológico (AP) confirmou adenocarcinoma mucinoso; citologia oncótica urinária negativa para células neoplásicas. O paciente submeteu-se, ainda no pré-operatório, à colonoscopia, sem alterações significativas.

Realizou-se cistectomia parcial aberta, com ressecção em bloco do úraco, incluindo o umbigo, fáscia posterior do músculo retoabdominal, peritônio adjacente, gordura perivesical e linfadenectomia ilíaco-obturatória. O AP da peça cirúrgica evidenciou adenocarcinoma mucinoso, medindo $7,5 \mathrm{~cm}$, infiltrando a parede muscular vesical e 0 úraco até o tecido adiposo; linfonodos, margens cirúrgicas e pele livres de comprometimento neoplásico; estadiamento de Sheldon IIIA e Henly/Mayo II.

Não foi indicado tratamento adjuvante para o paciente. No seguimento pós-operatório, após 12 meses da cirurgia, em cistoscopia de controle, encontrou-se recidiva tumoral em cúpula e a biópsia confirmou tratar-se de adenocarcinoma invasivo, moderadamente diferenciado. $\mathrm{O}$ indivíduo foi, então, submetido à cistoprostatectomia radical, com reconstrução urinária incontinente à Bricker. O AP foi conclusivo: adenocarcinoma mucinoso medindo $4 \mathrm{~cm}$ no maior eixo, infiltrando toda a espessura da parede até o tecido adiposo adjacente; infiltrações angiolinfática e perineural não foram detectadas; margens, próstata e vesículas seminais estavam livres de comprometimento neoplásico.

O paciente apresentou evolução pós-operatória insatisfatória (não considerada consequência direta do adenocarcinoma). Necessitou de reabordagens cirúrgicas para correção de infarto segmentar mesentérico, fístula urinária e entérica. Evoluiu a óbito.

\section{Caso clínico 2}

Paciente do sexo feminino, 55 anos de idade, com história de hematúria macroscópica associada à infecção do trato urinário. Ex-tabagista, hipertensa, sobrepeso (IMC $29,5 \mathrm{~kg} / \mathrm{m}^{2}$ ). A ultrassonografia (US) evidenciou espessamento em cúpula e formação nodular de 3,2 × 2,4 ×2,8 cm, vascularizada ao doppler. Submetida à cistoscopia e à RTU de lesão vesical, de cerca de $4 \mathrm{~cm}$, em cúpula, vegetante, papilífera e com franjas longas; citologia oncótica urinária negativa para células neoplásicas.

Diagnóstico imunopatológico com perfil imuno-histoquímico que favorecia carcinoma (painel imuno-histoquímico utilizado: Mx CK20 - 1:100 RA [Dako] - positivo; MCK7 - 1:100 RA [Invitrogen] - positivo; Mx CA125 - 1:50 RA [DBS] - negativo; Mx AE1/AE3 - 1:400 RA [Cell Marque] - positivo) e sugeria pesquisar possíveis sítios primários: ovários e bexiga.

Após 45 dias, realizaram-se novos exames. Endoscopia digestiva alta e US transvaginal sem alterações significativas. TC de abdômen com imagem de massa tumoral sólida, de $5 \times 4 \mathrm{~cm}$, em cúpula vesical. Foi, então, submetida à nova RTU de bexiga, encontrando lesão sólida em cúpula; restante da mucosa vesical livre. O AP indicava adenocarcinoma mucinoso com células em anel de sinete e camada muscular infiltrada pela neoplasia (Figura 2). Sem evidências de lesões uroteliais.

Realizada a cistectomia parcial videolaparoscópica, com ressecção em bloco do úraco, incluindo o umbigo, fáscia posterior do músculo retoabdominal, peritônio adjacente e gordura perivesical. O AP mostrou adenocarcinoma mucinoso, medindo $3 \mathrm{~cm}$ em seu maior eixo, invadindo o tecido adiposo adventicial e margens; pele e tecido subcutâneo livres de comprometimento neoplásico; estadiamento de Sheldon IIIA e Henly/Mayo II. 


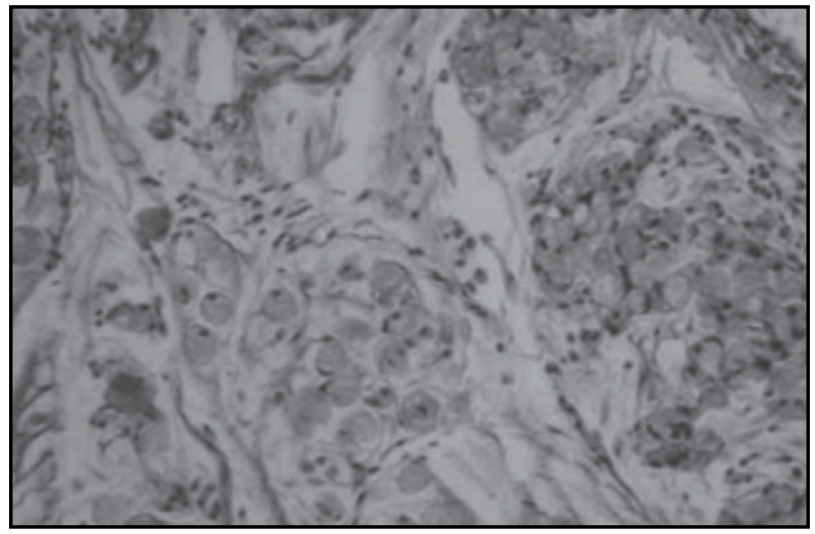

Figura 1 - Adenocarcinoma mucinoso com células em anel de sinete (HE, 400x) HE: hematoxilina e eosina.

Seguimento tomográfico e cistoscópico, após 12 meses, não mostrou recidiva tumoral. $\mathrm{O}$ serviço de oncologia não indicou quimioterapia adjuvante. Encontra-se em regime de vigilância ativa.

\section{Discussão}

O úraco origina-se da cloaca fetal durante a quinta semana e comunica o ápice da bexiga fetal primitiva ao alantoide. É constituído de três camadas: (i) luminal composta de epitélio cuboide ou transicional; (ii) tecido conjuntivo submucoso intermediário; e (iii) camada muscular externa. A partir do quarto mês do desenvolvimento fetal normal, a bexiga migra até a pelve e o lúmen do canal é, progressivamente, obliterado. No adulto, normalmente, consiste em um cordão fibroso, com 5 a $10 \mathrm{~cm}$; ligamento umbilical mediano, localizado entre a fáscia transversalis, e peritônio, no espaço de Retzius ${ }^{(1,3)}$. Autópsias em adultos mostram cerca de $30 \%$ de presença de lúmen microscópico. Essa falha de obliteração proporciona uma variedade de anomalias, como úraco patente, cisto de úraco, divertículo uracovesical e a formação de neoplasias benignas e malignas ${ }^{(9)}$.

A despeito de a mucosa uracal ser composta por células transicionais, o tipo histológico neoplásico mais comum é o adenocarcinoma mucinoso, fato que se deve à provável metaplasia glandular do epitélio transicional, com subsequente malignização ${ }^{(6,9)}$. Ou, como sugerem outros autores, a origem glandular da neoplasia seria em razão da origem comum da bexiga e do reto, a partir da cloaca, e a inclusão no úraco de mucosa entérica residual secretora de muco(9). Outros tipos histológicos menos comuns são os carcinomas uroteliais de células escamosas, de pequenas células e de células em anel de sinete ${ }^{(1)}$.
Em comparação com adenocarcinomas não úraco, os adenocarcinomas de úraco ocorrem em pacientes mais jovens (idade média 56 versus 69 anos, $p<0,0001$ ) e muIheres ( $45 \%$ versus $36 \%, p=0,02$ ). São lesões com menor probabilidade de serem de alto grau (35\% versus $66 \%$, $p<0,001)$, mas com maior probabilidade de apresentarem metástase a distância $(30 \% \text { versus } 15 \%, p<0,001)^{(10)}$.

Hematúria é o sintoma mais comum, presente em cerca de $80 \%$ dos indivíduos. Dor suprapúbica, sintomas irritativos do trato urinário inferior, mucosúria, massa infraumbilical palpável e infecção do trato urinário também podem ocorrer ${ }^{(1)}$.

Os dois principais tipos de sistemas de estadiamento do tumor de úraco são os de Sheldon ${ }^{(8)}$ (Tabela 1) e Henly(4) (Tabela 2). O estadiamento deste foi posteriormente modificado e simplificado pela clínica de Mayo ${ }^{(1)}$, contudo, ambos os sistemas predizem mortalidade de câncer específica.

\section{Tabela 1 Classificação de Sheldon ${ }^{(8)}$}

\begin{tabular}{|c|c|}
\hline Estadio & Definição \\
\hline Estadio I & Câncer de úraco confinado à mucosa \\
\hline Estadio II & $\begin{array}{l}\text { Câncer de úraco com invasão confinada } \\
\text { ao úraco }\end{array}$ \\
\hline Estadio IIIA & $\begin{array}{l}\text { Câncer de úraco local com extensão até } \\
\text { a bexiga }\end{array}$ \\
\hline Estadio IIIB & $\begin{array}{l}\text { Câncer de úraco local com extensão até } \\
\text { a parede abdominal }\end{array}$ \\
\hline Estadio IIIC & $\begin{array}{l}\text { Câncer de úraco local com extensão até } \\
\text { o peritônio }\end{array}$ \\
\hline Estadio IIID & $\begin{array}{l}\text { Câncer de úraco local com extensão } \\
\text { para outros órgãos, exceto bexiga }\end{array}$ \\
\hline Estadio IVA & Metástases para linfonodos \\
\hline Estadio IVB & Metástases para outros órgãos \\
\hline
\end{tabular}

\section{Tabela 2 Classificação de Henly /Mayo(1)}

\begin{tabular}{|c|c|}
\hline Estadio & Definição \\
\hline Estadio I & $\begin{array}{l}\text { Câncer de úraco confinado ao úraco e à } \\
\text { bexiga }\end{array}$ \\
\hline Estadio II & $\begin{array}{l}\text { Câncer de úraco além da parede } \\
\text { muscular do úraco e da bexiga }\end{array}$ \\
\hline Estadio III & $\begin{array}{l}\text { Câncer de úraco local com extensão } \\
\text { para linfonodos }\end{array}$ \\
\hline Estadio IV & $\begin{array}{l}\text { Câncer de úraco local com extensão } \\
\text { para outros órgãos e linfonodos não } \\
\text { regionais }\end{array}$ \\
\hline
\end{tabular}


A remoção cirúrgica é crucial para a sobrevivência e cura de pacientes com doença não metastática. A cistectomia parcial é, frequentemente, usada como técnica de eleição, pois, comparativamente, os resultados a longo prazo não diferem entre cistectomia radical e parcial ${ }^{(3,8)}$. Todavia, a questão sobre qual ato cirúrgico, se cistectomia parcial ou radical é mais apropriada para doença localizada, é difícil de ser respondida em razão da raridade da patologia ${ }^{(7)}$.

As vias de acesso cirúrgico, aberta ou laparoscópica, têm sido empregadas. A cistectomia parcial laparoscópica, em indivíduos cuidadosamente selecionados, é uma opção de terapia minimamente invasiva, factível e segura para esses pacientes ${ }^{(2)}$.

A adesão aos princípios cirúrgicos oncológicos, com dissecção pélvica ampliada, incluindo umbigo, tumor e todo o úraco, com margens negativas, cura a maioria dos pacientes com carcinoma de úraco localizado ${ }^{(5)}$.

As taxas de cura com cistectomia parcial oncológica chegam a $70 \%$ nos tumores clinicamente localizados e a $80 \%$ nos casos em que o tumor está confinado à peça cirúrgica ${ }^{(5)}$. Pacientes com doença confinada à bexiga, ao úraco e à gordura periuracal têm sobrevida, em cinco anos, de $88 \%$, ao passo que, nos casos em que existe invasão da cavidade peritoneal, não há sobrevida nesse período ${ }^{(5)}$.
Embora a maioria dos tumores de úraco invada a bexiga, eles são associados ao prognóstico ruim em razão do estadio avançado ao diagnóstico e ao crescimento tumoral extravesical, que favorece a recorrência local e a metástase a distância ${ }^{(5)}$. Séries da literatura reportam taxas de sobrevida livre de doença, após cirurgia, em cinco anos, de $11 \%$ a $55 \%$ para adenocarcinomas não úraco e de $27 \%$ a $61 \%$ para adenocarcinomas de úraco ${ }^{(1,4,5,8,10)}$.

Margem cirúrgica (odds ratio [OR] 4,7), alto grau (OR $3,6)$, linfonodo local positivo (OR 5,1), metástase ao diagnóstico (OR 3,3), estadio tumoral avançado (OR 4,8) e falha na umbilectomia (OR 3) foram associados à morte na análise univariada $(p>0,05)$. Somente grau e margem tumoral foram significantes na análise multivariada e são considerados os preditores mais significativos de sobrevida ${ }^{(1,5)}$.

Nenhum benefício quanto à sobrevida foi notado com a linfadenectomia e terapias adjuvantes ${ }^{(1)}$. Contudo, para outros autores a linfadenectomia parece ter valor prognóstico em pacientes selecionados. O status linfonodal torna-se mais importante à medida que o efeito da quimioterapia seja adquirido ${ }^{(5)}$. Cirurgia de salvação resultou em cura de $50 \%$ dos pacientes que apresentaram recorrência local. Nenhum tratamento efetivo foi identificado em indivíduos com doença metastática ${ }^{(1)}$.

\section{Referências}

1. ASHLEY, R. A. et al. Urachal carcinoma: clinicopathologic features and long-term outcomes of an aggressive malignancy. Cancer, v. 107, p. 712-20, 2006.

2. COLOMBO Jr, J. R. et al. Laparoscopic partial cystectomy for urachal and bladder cancer. Clinics, v. 63, p. 7314, 2008.

3. D'ADDESSI, A. et al. Adenocarcinoma of the urachus: radical or conservative surgery? A report of a case and a review of the literature. Eur J Surg Oncol, v. 24, p. 131-3, 1998.

4. HENLY, D. R.; Farrow, G. M.; Zincke, H. Urachal cancer: role of conservative surgery. Urology, v. 42, p. 635-9, 1993.

5. HERR H. W. et al. Urachal carcinoma: contemporary surgical outcomes. J Urol, v. 178, p. 74-8, discussion 78, 2007.
6. MUNICHOR M. et al. Mixed adenocarcinoma and neuroendocrine carcinoma rising in the urachus. Eur Urol, v. 28, p. 345-7, 1995.

7. SCABINI S. et al. Urachal tumour: case report of a poorly understood carcinoma. World J Surg Oncol, v. 7, n. 82, 2009.

8. SHELDON, C. A. et al. Malignant urachal lesions. J Urol, v. 131, p. 1-8, 1984.

9. THALI-SCHWAB, C. M.; WOODWARD, P. J.; WAGNER, B. J. Computed tomographic appearance of urachal adenocarcinomas: review of 25 cases. Eur Radiol, v. 15, p. 79-84, 2005.

10. WRIGHT, J. L. et al. Differences in survival among patients with urachal and nonurachal adenocarcinomas of the bladder. Cancer, v. 107, p. 721-8, 2006. 